\title{
INHERITANCE OF SYMMETRY FOR POSITIVE SOLUTIONS OF SEMILINEAR ELLIPTIC BOUNDARY VALUE PROBLEMS
}

\section{TRANSMISSION DES PROPRIÉTÉS DE SYMÉTRIE POUR DES SOLUTIONS POSITIVES DES PROBLÈMES ELLIPTIQUES SEMI-LINÉAIRES}

\author{
Bernd KAWOHL ${ }^{\text {a }}$, Guido SWEERS ${ }^{\mathrm{b}}$ \\ a Mathematical Institute, University of Cologne, 50923 Cologne, Germany \\ ${ }^{\mathrm{b}}$ Applied Math. Analysis, Delft University of Technology, PO Box 5031, 2600GA Delft, The Netherlands
}

Received 5 May 2001, revised 29 November 2001

ABSTRACT. - The paper addresses symmetry results for positive solutions of semilinear elliptic differential equations on a class of non-convex symmetrical domains. An example in two dimensions is the star of David. The moving plane method just shows that solutions coincide on three alternate corners of the star. We will show that the solution is symmetric with respect to remaining reflections, that is, the solution will have the full symmetry. To obtain such type of result, even for domains in higher dimensions, we use a variant of the sliding-method and the maximum principle for domains with small measure.

(C) 2002 L'Association Publications de l'Institut Henri Poincaré. Published by Elsevier B.V. All rights reserved

RÉSUMÉ. - Des résultats concernant la symétrie des solutions positives d' équations elliptiques semilinéaires sur une classe de domaines symétriques nonconvexes sont établis. Un exemple en dimension deux est l' étoile de David. La méthode des plans mobiles dans sa forme habituelle permet de montrer le symétrie de telles solutions seulement par rapport à trois axes. Nous sommes en mesure de prouver la symétrie par rapport au centre et donc la symétrie totale. Pour obtenir ce type de résultat, même pour des domaines en dimension supérieure à deux, nous utilisons la méthode des balayages et le principe du maximum pour les domaines de mesure petite.

(C) 2002 L'Association Publications de l'Institut Henri Poincaré. Published by Elsevier B.V. All rights reserved

E-mail addresses: kawohl@math.uni-koeln.de (B. Kawohl), g.h.sweers@its.tudelft.nl (G. Sweers). 


\section{Introduction}

A basic question for positive solutions of the elliptic boundary value problem

$$
\begin{cases}-\Delta u=f(u) & \text { in } \Omega \\ u=0 & \text { on } \partial \Omega\end{cases}
$$

with $\Omega \subset \mathbb{R}^{N}$ bounded and $f$ Lipschitz, is the following:

Is the symmetry of the domain passed on to the symmetry of the solution?

For $\Omega=B$, a ball, this question is solved by Gidas, Ni and Nirenberg in [9]: any positive solution of (1) with $\Omega=B$ is radially symmetric. The same question on other domains may not give a positive answer. For domains such as an annulus (see [4], [11, Theorem 3]), or a symmetric starshaped bar-less dumb-bell (see [6]), there are nonlinearities with positive solutions that do not reflect the symmetry of the domain.

The result of [9] can be used to obtain symmetry of positive solutions on domains with a reflection symmetry that are more general than just a ball. The additional condition necessary for the argument of [9] is that the domain is also convex in the direction perpendicular to the plane of symmetry. The combination of these two conditions is known as Steiner-symmetry. And indeed, then the method of moving planes, with the extension of [2], implies that any positive solution is symmetric and even symmetrically (strictly) decreasing with respect to that plane. As a direct consequence it follows that positive solutions on regular polygons inherit the symmetry of that polygon. For example, if the domain is a square, $\Omega=Q:=\left\{x \in \mathbb{R}^{2} ;\left|x_{i}\right|<1, i=1,2\right\}$, then

$$
u\left(x_{1}, x_{2}\right)=u\left(x_{2}, x_{1}\right)=u\left(-x_{2}, x_{1}\right) \quad \text { for all } x \in Q .
$$

Obviously, a convex domain $\Omega$ is Steiner-symmetric in all of its planes of symmetry, and one finds that the solution has the full symmetry. But one can do with less. We will prove that there are many cases where Steiner-symmetry in some directions is sufficient for any positive solution to inherit the full symmetry. A typical example in $\mathbb{R}^{2}$ is the star of David. Although it has 6 axes of symmetry, it is Steiner-symmetric in only 3 directions. Hence by using the arguments as in [9] one finds

$$
u\left(x_{1}, x_{2}\right)=u\left(-x_{1}, x_{2}\right)=u\left(R_{2 \pi / 3}\left(x_{1}, x_{2}\right)\right) \quad \text { for all } x \in \text { S.o.D. }
$$

(as in Fig. 1), where $R_{2 \pi / 3}$ is the rotation around the center with angle $2 \pi / 3$. The argument does not show that the solution is invariant under a rotation $R_{\pi / 3}$. In other

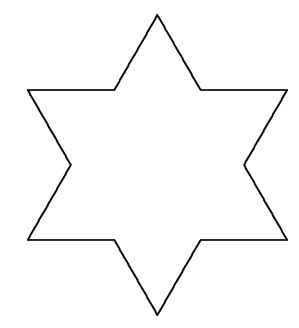

Fig. 1. Star of David. 
words, if $u$ is a solution, then, a priori, $u \circ R_{\pi / 3}$ could be a different solution. We will supply an argument that allows us to compare a solution with its reflection in a nonSteiner direction. As a consequence positive solutions on domains such as the Star of David have the full symmetry. The question whether or not positive solutions on the hexagonal and pentagonal star are symmetric was mentioned in [15].

\section{Main results}

First let us recall the definition of Steiner-symmetry:

Definition 1. - A domain $\Omega \subset \mathbb{R}^{N}$ is called Steiner-symmetric with respect to the (hyper-)plane $x_{1}=0$, if it is convex and symmetric in the $x_{1}$-direction, in other words, if for every $\left(x_{1}, x^{\prime}\right):=\left(x_{1}, x_{2}, \ldots, x_{N}\right) \in \Omega$,

$$
\left[\left(x_{1}, x^{\prime}\right),\left(-x_{1}, x^{\prime}\right)\right]:=\left\{\left(t, x^{\prime}\right) ;|t| \leqslant\left|x_{1}\right|\right\} \subset \Omega .
$$

DEFINITION 2. - A function $u: \Omega \mapsto \mathbb{R}$ is called Steiner-symmetric with respect to the plane $x_{1}=0$, if all of its level sets $\Omega_{c}:=\{x \in \Omega: u(x)>c\}$ are Steiner-symmetric with respect to the plane $x_{1}=0$.

To present our results we distinguish two cases. First we consider domains which are invariant under rotations about one axis, and reflections about a hyperplane containing this axis, later those which are invariant under rotations about multiple axes.

\subsection{Symmetry of $\Omega \subset \mathbb{R}^{N}$ under rotations and reflections for the first two coordinates}

The domains that we consider in this section are invariant under the rotation $R_{\pi / n}$ in the $\left(x_{1}, x_{2}\right)$-plane of angle $\pi / n$ and a reflection across the plane $x_{1}=0$. Here $n$ is some fixed number in $\mathbb{N}^{+}$. Domains with this symmetry include the star of David and domains in $\mathbb{R}^{3}$ as in Fig. 2. The corresponding algebraic group is known as the dihedral group $\mathcal{D}_{2 n}$. We exclude geometries that are symmetric under odd rotations such as the pentagonal "red" star or the tetrapod in $\mathbb{R}^{3}$ from Fig. 3.

THEOREM 3. - Let $n \in \mathbb{N}^{+}$and suppose that $\Omega \subset \mathbb{R}^{N}$ is invariant under rotation by $\pi / n$ in the $\left(x_{1}, x_{2}\right)$-plane. Moreover, assume that $\Omega$ is Steiner-symmetric with respect to the plane $x_{1}=0$. Then any positive solution $u \in C(\bar{\Omega}) \cap C^{2}(\Omega)$ of (1) satisfies

$$
u\left(x_{1}, x^{\prime}\right)=u\left(-x_{1}, x^{\prime}\right)=u\left(R_{\pi / n}(x)\right) \quad \text { for all } x=\left(x_{1}, x^{\prime}\right) \in \Omega .
$$
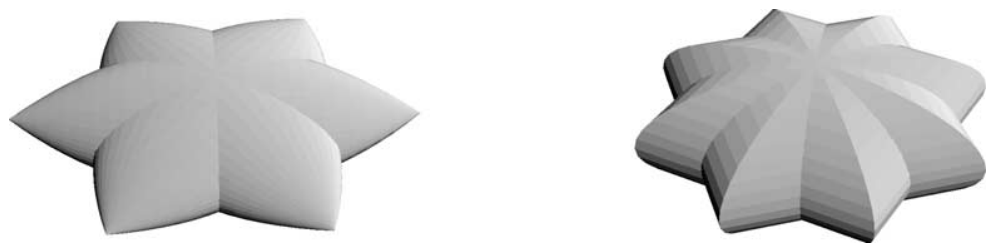

Fig. 2. Invariant under rotation/reflection with a Steiner-symmetry. 

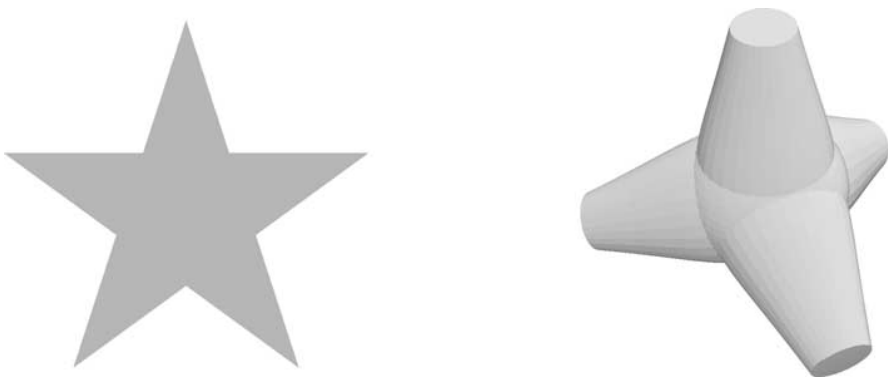

Fig. 3. Odd order rotation-symmetries, no Steiner-symmetry.

\subsection{Invariance of $\Omega$ under cubic symmetry}

Representations of this group are well known: the rotation and reflection symmetries of both the cube and the regular octahedron. The corresponding point group is denoted by $\mathbb{O}_{h} . \mathbb{O}_{h} \simeq \mathbb{O} \times \mathbb{Z}_{2}$ where the octahedral group $\mathbb{O}$ corresponds to the rotations of the regular octahedron and $\mathbb{Z}_{2}$ with the reflection in the point $(0,0,0)$. Since we are interested in geometric, and not algebraic questions, we have to define the respective group actions in relation to a fixed direction for the Steiner-symmetry. For algebraic properties of symmetry groups we refer to [1] and [13]. For regular polyhedra see [5].

- $\mathcal{R}_{1}$ represents $\mathbb{O}_{h}$ as generated by the rotations of angle $\pi / 2$ about every cartesian axis, and the reflections in the planes $x_{i}=0, i=1,2,3$.

- $\mathcal{R}_{2}$ represents $\mathbb{O}_{h}$ as generated by the rotation of angle $\pi / 2$ about the $x_{3}$-axis, the rotations of angle $\pi / 2$ about lines through zero perpendicular to the planes $x_{1}=x_{2}$ respectively $x_{1}=-x_{2}$, and the reflections in the planes $x_{i}=0, i=1,2,3$.

See [13, p. 74] to find that $\mathbb{O}_{h}$ can be generated by three elements.

In this subsection we restrict our attention to two model cases of nonconvex domains. The first one is a three-dimensional star like the one in Fig. 4(left), whose corners point into the direction of the axes, just like the normal vectors on a cube. This domain is invariant under elements $T \in \mathcal{R}_{1}$. It is Steiner-symmetric with respect to the planes $x_{i}=0, i=1,2,3$. By the method of moving planes any positive solution of (1) is identical in opposite points, i.e.

$$
u\left(x_{1}, x_{2}, x_{3}\right)=u\left( \pm x_{1}, \pm x_{2}, \pm x_{3}\right) \quad \text { for all } x \in \Omega .
$$

However, a priori $u(x)=u\left(x_{1}, x_{2}, x_{3}\right), v(x)=u\left(x_{2}, x_{1}, x_{3}\right)$ and $w(x)=u\left(x_{1}, x_{3}, x_{2}\right)$ could all be different from each other. Therefore the following result is new.

COROLlaRY 4. - Let $\Omega$ be invariant under $\mathcal{R}_{1}$ and Steiner symmetric with respect to $x_{1}=0$, such as in Fig. 4(left). Then any positive solution $u \in C(\bar{\Omega}) \cap C^{2}(\Omega)$ of (1) satisfies

$$
u\left(x_{1}, x_{2}, x_{3}\right)=u\left(T\left(x_{1}, x_{2}, x_{3}\right)\right) \quad \text { for all } x=\left(x_{1}, x_{2}, x_{3}\right) \in \Omega \text { and } T \in \mathcal{R}_{1} .
$$

The second model domain is constructed as follows. If we glue a regular tetrahedron on each face of a regular octahedron, we obtain a stellated octahedron as in Fig. 4(right). 

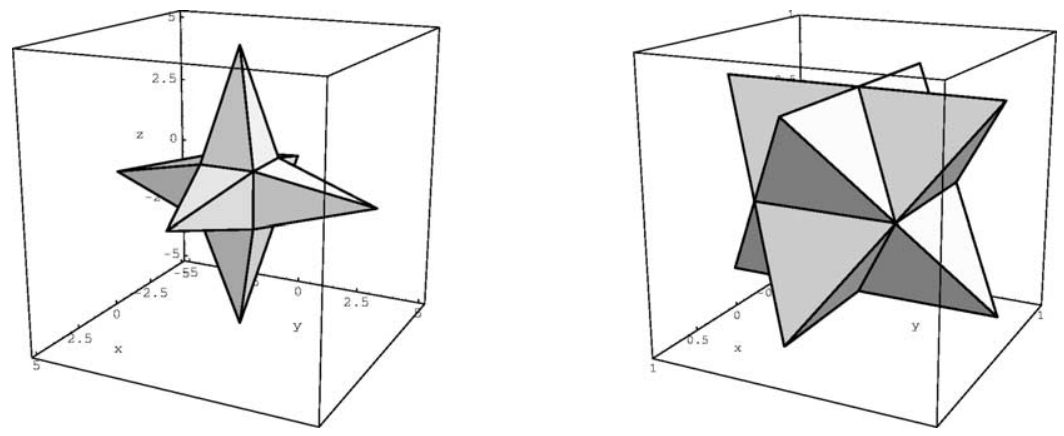

Fig. 4. (Left) A stellated cube and (right) Kepler's Stella Octangula, which are invariant under $\mathcal{R}_{1}$, respectively $\mathcal{R}_{2}$. The symmetries of both bodies give rise to the same algebraic group $\mathbb{O}_{h}$.

This polyhedron is known as Kepler's Stella Octangula. One can also look at it as a combination of two large tetrahedrons. It is invariant under the elements of $\mathcal{R}_{2}$. From the moving plane method we may conclude that

$$
u\left(x_{1}, x_{2}, x_{3}\right)=u\left( \pm x_{1}, \pm x_{2}, x_{3}\right)=u\left( \pm x_{2}, \pm x_{1},-x_{3}\right) \text { for all } x \in \Omega .
$$

However, a priori $u(x)=u\left(x_{1}, x_{2}, x_{3}\right)$ and $v(x)=u\left(x_{1}, x_{2},-x_{3}\right)$ could be different from each other. This is ruled out by the following result.

COROLlaRY 5. - Let $\Omega$ be invariant under $\mathcal{R}_{2}$ and Steiner symmetric with respect to $x_{1}=0$, such as in Fig. 4(right). Then any positive solution $u \in C(\bar{\Omega}) \cap C^{2}(\Omega)$ of (1) satisfies

$$
u\left(x_{1}, x_{2}, x_{3}\right)=u\left(T\left(x_{1}, x_{2}, x_{3}\right)\right) \quad \text { for all } x=\left(x_{1}, x_{2}, x_{3}\right) \in \Omega \text { and } T \in \mathcal{R}_{2} .
$$

\section{Proofs}

Proof of Theorem 3. - Although the theorem above holds for general dimensions for the sake of simplicity we will illustrate the proof by the two-dimensional star of David.

Using the Steiner-symmetry with respect to the plane $x_{1}=0$ and generalizations of the result by Gidas, Ni and Nirenberg [9] to nonsmooth domains (see [2,3] and [8, Theorem 3.3]) one finds that

$$
\begin{gathered}
u\left(x_{1}, x^{\prime}\right)=u\left(-x_{1}, x^{\prime}\right) \quad \text { for all }\left(x_{1}, x^{\prime}\right) \in \Omega, \\
\frac{\partial}{\partial x_{1}} u\left(x_{1}, x^{\prime}\right)<0 \quad \text { for all }\left(x_{1}, x^{\prime}\right) \in \Omega \text { with } x_{1}>0 .
\end{gathered}
$$

Similar claims are true with respect to rotations in the $\left(x_{1}, x_{2}\right)$-plane by $2 \pi / n$. This does not yield the full symmetry as claimed in the theorem, because we are not allowed to draw conclusions for a rotation by $\pi / n$ yet. Comparing with the star of David we only find that the value of $u$ is identical at identical symbols in Fig. 5 . 


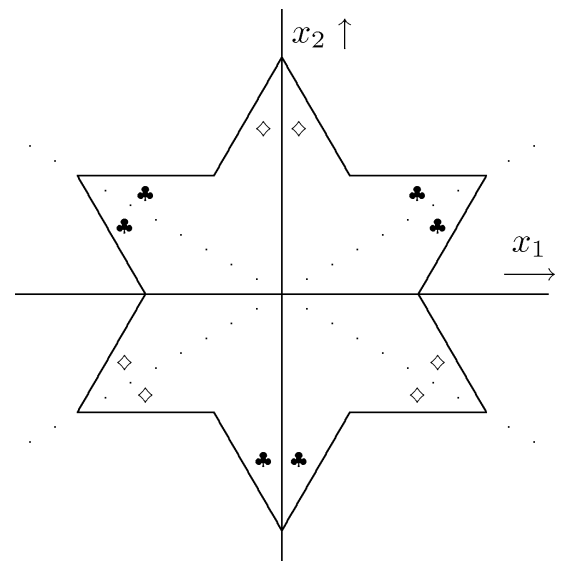

Fig. 5. Symmetries due to the moving plane method.

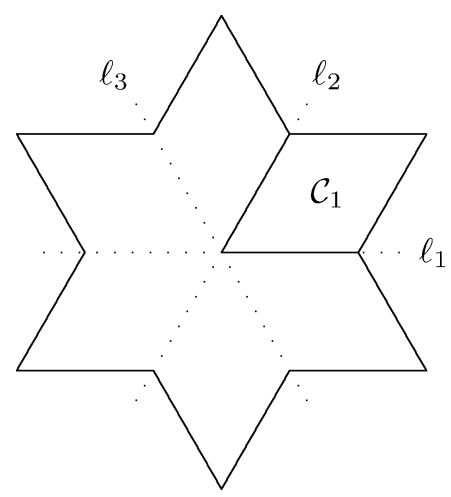

Fig. 6. Notations used in the proof.

In the next step we will compare $u$ with its reflection $v$ in the plane $x_{2}=0$. Because of the already known symmetries of $u$ this reflection coincides with a rotation by $\pi / n$. Even if these two functions $u$ and $v$ are not identical we find by the symmetry from the moving plane argument above, that

$$
u(x)=v(x) \text { for all } x \in \ell_{i}, i=1, \ldots, n,
$$

where the $\ell_{i}, i=1, \ldots, n$, denote the remaining planes of symmetry of $\Omega$ (see Fig. 6).

We intend to show that $u$ and $v$ coincide everywhere in $\Omega$. To do so we proceed by contradiction and consider one of the components of $\Omega \backslash \bigcup_{i=1}^{n} \ell_{i}$. To fix the argument we choose the one between $\ell_{1}$ and $\ell_{2}$ on the right half space and denote it by $\mathcal{C}_{1}$. It will suffice to show that $u$ and $v$ coincide on $\mathcal{C}_{1}$. Without loss of generality we may assume that there is $y \in \mathcal{C}_{1}$ such that $u(y)<v(y)$.

On this component we will apply a sweeping argument. See [12] for a statement of the so-called sweeping principle. Instead of joining the solution and a reflected copy as in [9] we start with $u$ and a copy of $v$ which is shifted to the left. Then we move $v$ in the $x_{1}$-direction 'under' $u$ until it touches $u$ from below somewhere on $\mathcal{C}_{1}$. We set

$$
v\left(t ; x_{1}, x^{\prime}\right):=v\left(x_{1}+t, x^{\prime}\right) \quad \text { for } x=\left(x_{1}, x^{\prime}\right) \in \Omega,
$$

and denote by $\Omega(t)$ the support of $v(t ; \cdot)$. Note that for large positive $t$ the set $\Omega(t)$ lies to the left of $\Omega$. Suppose that $u \neq \equiv v(0 ; \cdot)$.

Now let us remind that, since $x_{1}>0$ for $x \in \mathcal{C}_{1}$, the moving plane argument in the first step of this proof gives us that both $u$ and $v$ are strictly decreasing on $\mathcal{C}_{1}$ in the $x_{1}$-direction:

$$
\begin{array}{ll}
\frac{\partial}{\partial x_{1}} u\left(x_{1}, x^{\prime}\right)<0 & \text { for all }\left(x_{1}, x^{\prime}\right) \in \mathcal{C}_{1}, \\
\frac{\partial}{\partial x_{1}} v\left(x_{1}, x^{\prime}\right)<0 & \text { for all }\left(x_{1}, x^{\prime}\right) \in \mathcal{C}_{1} .
\end{array}
$$


Hence we have for $0 \leqslant t_{1}<t_{2}$ that

$$
v\left(t_{2} ; x\right)<v\left(t_{1} ; x\right) \quad \text { for } x \in \mathcal{C}_{1} \cap \Omega\left(t_{1}\right) \cap \Omega\left(t_{2}\right) .
$$

Setting $A(t):=\left\{x \in \mathcal{C}_{1} \cap \Omega(t) ; u(x)<v(t ; x)\right\}$, the previous observation yields that $A\left(t_{2}\right) \subset A\left(t_{1}\right)$ for $0 \leqslant t_{1}<t_{2}$. Since we have assumed that $u(y)<v(0 ; y)$ it follows that $A(0)$ is nonempty. Moreover, since $A(t)$ is empty for $t$ large and since the $A(t)$ are open for all $t$, there exists a smallest $t_{0}>0$ such that $A(t)$ is empty for $t \geqslant t_{0}$. Next we fix a point $z$ where $u$ and $v\left(t_{0} ; \cdot\right)$ 'touch'. Indeed by the minimality of $t_{0}$, for every $n \in \mathbb{N}^{+}$ there exists a $z_{n}$ in $A\left(t_{0}-\frac{1}{n}\right)$, and this sequence has a convergent subsequence with limit $z$. We assume without loss of generality that $z_{n} \rightarrow z$.

Two possibilities are conceivable: either $z \in \overline{\mathcal{C}_{1}} \cap \Omega\left(t_{0}\right)$ or $z \in \overline{\mathcal{C}_{1}} \cap \partial \Omega\left(t_{0}\right)$. If $z \in$ $\overline{\mathcal{C}_{1}} \cap \Omega\left(t_{0}\right)$, then $z \in \mathcal{C}_{1} \cap \Omega\left(t_{0}\right)$ because $t_{0}>0$ and

$$
v\left(t_{0} ; x\right)<v(0 ; x)=u(x) \quad \text { for all } x \in \partial \mathcal{C}_{1} \cap \Omega\left(t_{0}\right) .
$$

We find that

$$
\begin{cases}-\Delta\left(u-v\left(t_{0} ; \cdot\right)\right)=g(x) \cdot\left(u-v\left(t_{0} ; \cdot\right)\right) & \text { in } \mathcal{C}_{1} \cap \Omega\left(t_{0}\right) \\ \left(u-v\left(t_{0} ; \cdot\right)\right) \geqslant 0 & \text { in } \mathcal{C}_{1} \cap \Omega\left(t_{0}\right)\end{cases}
$$

where the Lipschitz condition for $f$ implies that $g$, defined by

$$
g(x):=\int_{0}^{1} f^{\prime}(\theta u(x)+(1-\theta) v(x)) \mathrm{d} \theta,
$$

is bounded. By the strong maximum principle one finds that either $u \equiv v\left(t_{0} ; \cdot\right)$ or $u>v\left(t_{0} ; \cdot\right)$ in $\mathcal{C}_{1} \cap \Omega\left(t_{0}\right)$, which both give a contradiction.

The case $z \in \overline{\mathcal{C}_{1}} \cap \partial \Omega\left(t_{0}\right)$ remains. Moreover, since we already excluded $z \in \overline{\mathcal{C}_{1}} \cap$ $\Omega\left(t_{0}\right)$, we may assume that

$$
u>v\left(t_{0} ; \cdot\right) \quad \text { in } \overline{\mathcal{C}_{1}} \cap \Omega\left(t_{0}\right) .
$$

Now we will use the maximum principle for small domains. See [3] or [8, Th. 2.19]:

There exists $\delta=\delta\left(n,\left\|c^{+}\right\|_{\infty}\right.$, $\left.\operatorname{diam}(D)\right)$, such that if the Lebesgue measure of $D$ satisfies $|D|<\delta$, then a solution of $-\Delta u \geqslant c u$ in $D$ and $u \geqslant 0$ on $\partial D$, is nonnegative.

Fix $\delta=\delta\left(n, L_{f}, \operatorname{diam}\left(\mathcal{C}_{1}\right)\right)$ where $L_{f}$ is the Lipschitz constant of $f$. Next we choose an open neighborhood $\mathcal{O}$ of $\overline{\mathcal{C}_{1}} \cap \partial \Omega\left(t_{0}\right)$ such that the Lebesgue measure of $\mathcal{N}_{1}:=$ $\mathcal{O} \cap \overline{\mathcal{C}_{1}} \cap \Omega\left(t_{0}\right)$ satisfies $\left|\mathcal{N}_{1}\right|<\frac{1}{2} \delta$. On $\mathcal{K}:=\left\{x \in \overline{\mathcal{C}_{1}} \cap \Omega\left(t_{0}\right) ; x \notin \mathcal{N}_{1}\right\}$ there is $\varepsilon>0$ such $u \geqslant v\left(t_{0} ; \cdot\right)+\varepsilon$. Hence by continuity there exists $t_{1}<t_{0}$ such that

$$
u \geqslant v\left(t_{1} ; \cdot\right) \quad \text { on } \mathcal{K} .
$$

The number $t_{1}$ can be choosen sufficiently close to $t_{0}$ such that $\mathcal{N}_{2}:=\overline{\mathcal{C}_{1}} \cap \Omega\left(t_{1}\right) \backslash \overline{\Omega\left(t_{0}\right)}$ satisfies $\left|\mathcal{N}_{2}\right|<\frac{1}{2} \delta$. 


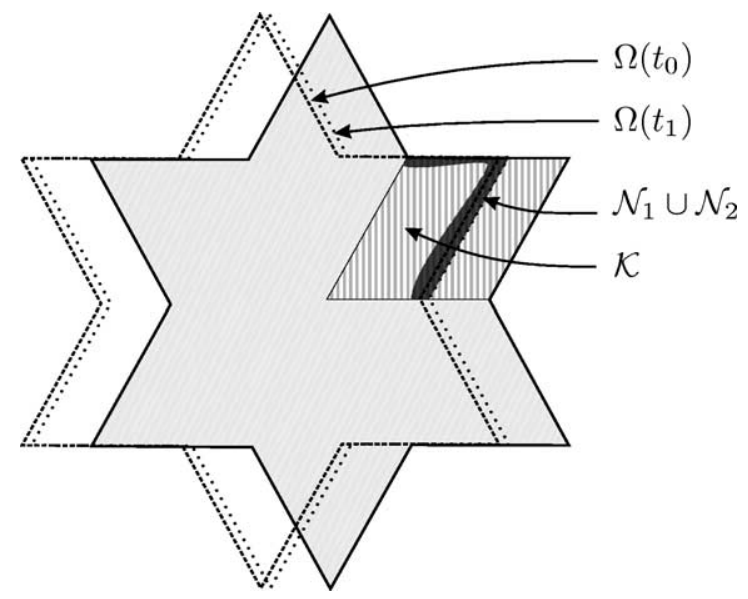

Fig. 7. Notations for the sliding method.

One may conclude by noting that $\partial\left(\mathcal{N}_{1} \cup \mathcal{N}_{2}\right) \subset \partial \mathcal{K} \cup \partial \Omega\left(t_{1}\right)$ implies $u \geqslant v\left(t_{1} ; \cdot\right)$ on $\partial\left(\mathcal{N}_{1} \cup \mathcal{N}_{2}\right)$ and hence by the above maximum principle $u \geqslant v\left(t_{1} ; \cdot\right)$ in $\mathcal{N}_{1} \cup \mathcal{N}_{2}$. With (14) it follows that

$$
u \geqslant v\left(t_{1} ; \cdot\right) \quad \text { in } \mathcal{N}_{1} \cup \mathcal{N}_{2} \cup \mathcal{K} \supset \overline{\mathcal{C}_{1}} \cap \Omega\left(t_{1}\right),
$$

which contradicts the minimality of $t_{0}$.

Proof of Corollary 4. - To verify this corollary, one has to realize that Theorem 3 can be applied with respect to each cartesian plane $\left(x_{i}, x_{j}\right), i \neq j$, and that the star in Fig. 4(left) is Steiner symmetric with respect to each plane $x_{i}=0, i, j=1,2,3$.

Proof of Corollary 5. - The proof of this corollary is reduced to another application of Theorem 3, but the details are more delicate because of the star's geometry. We notice that $\Omega$ is Steiner symmetric with respect to the six planes $x_{1}=0, x_{2}=0, x_{1}+x_{2}+$ $\sqrt{2} x_{3}=0, x_{1}-x_{2}+\sqrt{2} x_{3}=0,-x_{1}+x_{2}+\sqrt{2} x_{3}=0$ and $-x_{1}-x_{2}+\sqrt{2} x_{3}=0$. From the moving plane method we may then conclude that

$$
u\left(x_{1}, x_{2}, x_{3}\right)=u\left( \pm x_{1}, \pm x_{2}, x_{3}\right)=u\left( \pm x_{2}, \pm x_{1},-x_{3}\right) \quad \text { for all } x \in \Omega .
$$

These results are illustrated by each of configurations in Fig. 8, in which analogously to Fig. 5, identical colors indicate symmetries due to the moving plane method. Moreover, Fig. 5 shows that under this symmetry the reflection in $x_{3}=0$ coincides with a rotation.

However, since $\Omega$ is reflection symmetric in $x_{3}$, but not Steiner symmetric with respect to $x_{3}=0$, a priori $u(x)=u\left(x_{1}, x_{2}, x_{3}\right)$ and $v(x)=u\left(x_{1}, x_{2},-x_{3}\right)$ could be different from each other. Moreover, this reflection cannot be generated from those that are used in the moving plane method. It is in this situation, that one can mimmick the proof of Theorem 3. 

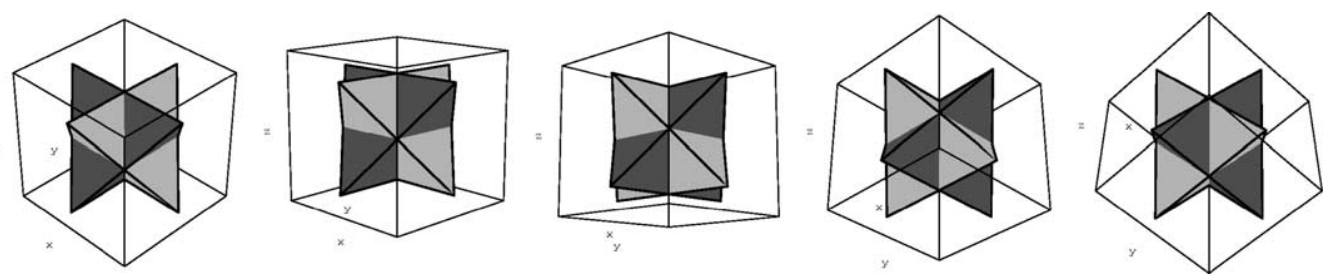

Fig. 8. Several views of the Stella Octangula.

\section{Related results and open problems}

In this paragraph we record some related observations.

Remark 6. - If problem (1) has a maximal positive solution $u$, then this $u$ inherits all the symmetries of $\Omega$.

Indeed, suppose that $\Omega$ is invariant under some group action $T$, but $u$ is not. Then $u(x)$ and $u(T x)$ are two different, non-ordered solutions, a contradiction to the maximality of $u$.

Remark 7. - If problem (1) has a positive solution $u$ and if $f(0)$ is positive, then (1) has at least one positive solution with all the symmetries of $\Omega$.

To see this, assume that $\Omega$ is invariant under a finite group $\mathcal{R}$ with elements $T_{i}, i=$ $1, \ldots, n$. Set $v(x):=\min \left\{u\left(T_{i} x\right) ; i=1, \ldots, n\right\}$. Then $v(x)$ is a positive symmetric supersolution of (1), while zero is a strict subsolution of (1). Therefore (1) has a maximal positive solution $w(x)$ in the interval $\left(0, v(x)\right.$ ], see [7]. Notice that $w\left(T_{i} x\right) \leqslant v\left(T_{i} x\right)=$ $v(x)$ for every $i=1, \ldots, n$. This and the maximality of $w$ imply $w(x)=w\left(T_{i} x\right)$ for all $T_{i} \in \mathcal{R}$.

For some domains the case $f(0)<0$ can be excluded on other grounds.

Remark 8. - If $\Omega \subset \mathbb{R}^{2}$ has an acute angle and if $f(0)<0$, then (1) cannot have a positive solution, see [14]. Therefore existence of a positive solution on the star of David implies that $f(0) \geqslant 0$.

Let us explicitly point out, that in the context of the above remarks there may also be nonsymmetric positive solutions of (1). This is the case for instance if $\Omega$ is an annulus, see $[4,10,11]$, or a dumb-bell [6]. To rule out any nonsymmetric positive solution of (1), as we have done in this paper, we need to assume Steiner symmetries, starshapedness and invariance of $\Omega$ under an appropriate reflection, i.e. a reflection across a hyperplane, with respect to which $\Omega$ is not Steiner-symmetric. However, we expect every positive solution of (1) to be symmetric on a larger class of domains, some of which are depicted in Fig. 9. To be more specific, we believe that any domain $\Omega$ with the property that all sets $\Omega_{d}:=\{x \in \Omega ; d(x, \partial \Omega)>d\}$ (for $d>0$ ) are starshaped with respect to zero, inherits any symmetry to any positive solution of (1).

Note that the first three domains in Fig. 9 fail to have Steiner-symmetries. Although the last one has such a Steiner-symmetry it is not in an appropriate direction. For this domain any positive solution $u\left(x_{1}, x_{2}, x_{3}\right)$ of (1) satisfies (4), but $u\left(x_{1}, x_{2}, x_{3}\right)$ might still differ from $u\left(x_{2}, x_{1}, x_{3}\right)$. 

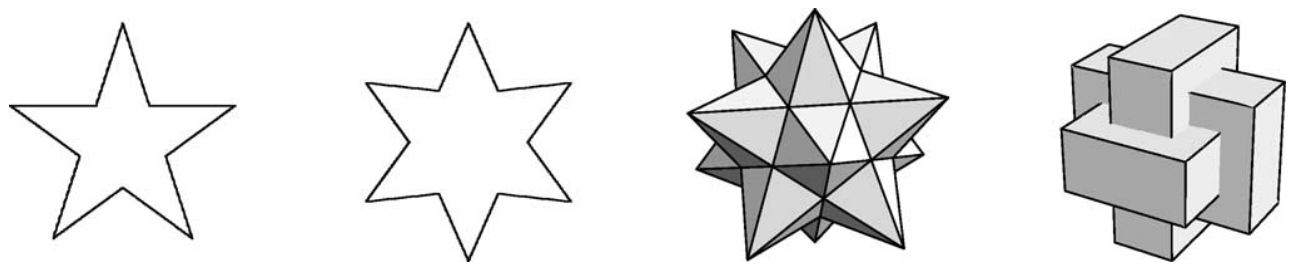

Fig. 9. Domains without appropriate symmetry. The third one is known as a Kepler-Poinsot polyhedron: the 'small stellated dodecahedron'.

Remark 9. - Finally we should mention that our main result, Theorem 3, remains true if the semilinear differential equation in (1) is replaced by a quasilinear one such as $-\operatorname{div}(a(u,|\nabla u|) \nabla u)=f(u,|\nabla u|)$, with $a$ and $f$ satisfying suitable assumptions.

\section{REFERENCES}

[1] S.L. Altmann, P. Herzig, Point-Group Theory Tables, Clarendon Press, Oxford, 1994.

[2] H. Berestycki, L. Nirenberg, Monotonicity, symmetry and antisymmetry of solutions of semilinear elliptic equations, J. Geom. Phys. 5 (1988) 237-275.

[3] H. Berestycki, L. Nirenberg, On the method of moving planes and the sliding method, Bol. Soc. Brasil. Mat. (N.S.) 22 (1991) 1-37.

[4] C.V. Coffman, A nonlinear boundary value problem with many positive solutions, J. Differential Equations 54 (1984) 429-437.

[5] H.S.M. Coxeter, Regular Polytopes, Dover Publications, New York, 1973.

[6] E.N. Dancer, The effect of the domain shape on the number of positive solutions of certain nonlinear equations, J. Differential Equations 74 (1988) 120-156.

[7] E.N. Dancer, G. Sweers, On the existence of a maximal weak solution for a semilinear elliptic equation, Differential Integral Equations 2 (1989) 533-540.

[8] L.E. Fraenkel, An Introduction to Maximum Principles and Symmetry in Elliptic Problems, Cambridge Tracts in Mathematics, Vol. 128, Cambridge University Press, Cambridge, 2000.

[9] B. Gidas, W.M. Ni, L. Nirenberg, Symmetry and related properties via the maximum principle, Comm. Math. Phys. 68 (1979) 209-243.

[10] B. Kawohl, A geometric property of level sets of solutions to semilinear elliptic Dirichlet problems, Applicable Anal. 16 (1983) 229-233.

[11] B. Kawohl, Symmetry results for functions yielding best constants in Sobolev-type inequalities, Discrete Continuous Dynam. Systems 6 (2000) 683-690.

[12] A. McNabb, A. Strong comparison theorems for elliptic equations of second order, J. Math. Mech. 10 (1961) 431-440.

[13] R. McWeeny, Symmetry, an Introduction to Group Theory and Its Applications, Pergamon Press, Oxford, 1963.

[14] G. Sweers, Semilinear elliptic problems on domains with corners, Comm. Partial Differential Equations 14 (1989) 1229-1247.

[15] G. Sweers, Lecture notes on maximum principles, December 2000, http://aw.twi.tudelft. $\mathrm{nl} / \sim \mathrm{sweers} / \mathrm{maxpr} /$ maxprinc.pdf. 